\title{
THE ROLE OF NEXT GENERATION SEQUENCING IN THE DIFFERENTIAL DIAGNOSIS OF CAROLI'S SYNDROME
}

\author{
Smolović $\mathrm{B}^{1}$, Muhović $\mathrm{D}^{1}$, Hodžić $\mathrm{A}^{2}$, Bergant $\mathrm{G}^{2}$, Peterlin $\mathrm{B}^{2, *}$ \\ *Corresponding Author: Professor Borut Peterlin, M.D., Ph.D., Department of Gynecology and \\ Obstetrics, Institute of Medical Genetics, Department of Gynecology and Obstetrics, University \\ Medical Centre Ljubljana, Šlajmerjeva Street 4, Ljubljana, Slovenia. Tel./Fax: +386-(0)1-540-1137. \\ E-mail: borut.peterlin@guest.arnes.si
}

\begin{abstract}
We report the case of a 41-year-old man on conservative treatment for more than 20 years for chronic renal insufficiency, chronic hepatitis and recurrent cholangitis. Following lengthy and extensive diagnostics, the differential diagnosis included primary sclerosing cholangitis and Caroli's disease (CD). To solve the diagnostic challenge, next generation sequencing (NGS) was performed to distinguish between the disorders possibly present in the patient. The diagnosis of CD became evident after two rare known pathogenic mutations were detected in the poly-ductin 1 (PKHD1) gene, c.370C $>$ T (p.Arg124Ter) and c.4870C $>$ T (p.Arg1624Trp). In this case, NGS was instrumental in solving the diagnostic challenge, allowing differentiation among the proposed genetic and nongenetic ethiologies.
\end{abstract}

Keywords: Caroli's syndrome (CS); Polyductin 1 (PKHD1) gene; Polycystic kidney disease.

\section{INTRODUCTION}

Caroli's disease (CD) was first described by Jacques Caroli in 1958 [1], as a rare congenital condition characterized by localized or diffuse, non obstructive, saccular or fusiform, multifocal segmental dilatation of the intrahepatic bile ducts. The disease prevalence is one case per $1,000,000$, however, with better imaging techniques, such

\footnotetext{
${ }^{1}$ Internal Clinic, Department of Gastroenterohepatology, Clinical Centre of Montenegro, Podgorica, Montenegro

${ }^{2}$ Clinical Institute of Medical Genetics, University Medical Centre Ljubljana, Ljubljana, Slovenia
}

as magnetic resonance cholangiography, $\mathrm{CD}$ appears to be more prevalent than previously reported $[2,3]$. Males and females are equally affected, and more than $80.0 \%$ of patients present before 30 years of age [4]. Recurrent cholangitis dominates the clinical course and is the principal cause of morbidity and mortality [5]. Two types of disease have been described: type 1 , a simple form or $\mathrm{CD}$ without hepatic fibrosis and type 2, a complex form or Caroli's syndrome (CS; also known as Grumbach's disease) with the presence of congenital hepatic fibrosis (CHF) $[3,6,7]$.

Caroli's disease (type 1) consists of pure segmental cystic dilatations of the intrahepatic bile ducts, without cirrhosis and portal hypertension. Caroli's disease can be diffuse, when it involves both lobes, or localized, when occuring in a single lobe. Increased incidence of biliary lithiasis, recurrent cholangitis, biliary abscesses and septicemia are all its potential complications $[4,6]$. Caroli's syndrome (type 2) presents a clinical syndrome that is a combination of CD (bouts of cholangitis, hepatolithiasis, and gallbladder stones) and those of CHF (portal hypertension). Caroli's syndrome is associated with hepatic fibrosis, or even cirrhosis, portal hypertension and oesophageal varices. Caroli's syndrome may be accompanied by chol-angiocarcinoma, pancreatic cyst and renal cystic disease $[4,6]$. These diseases have a close relationship with congenital kidney disorders, notably autosomal recessive polycystic kidney disease (ARPKD) [8,9]. Many investigators believe that the two types of CD are actually different stages of the same disease characterized by periportal fibrosis and ductal dilatation $[4,10]$.

Mutations in the polyductin 1 (PKHD1) gene, located on chromosome 6 (6p12.3-6p12.2), are responsible for $\mathrm{CD}$, and many causative mutations are known [11-13]. Only a few of these variants in PKHDI are responsible for ARPKD and CD, with a high interfamilial and in- 
trafamilial phenotypic variability $[9,11,12$,$] . We present$ a middle-aged patient, with very rare mutations in the PKHD1 gene, which led to CD with ARPKD that was, prior to NGS testing, misdiagnosed as primary sclerosing cholangitis (PSC).

\section{CASE PRESENTATION}

Patient Description. A 41-year-old man presented with fever, chills, fatigue, nausea, vomiting and pain in right upper abdominal quadrant. During physical examination, we observed a poor general state with a temperature of $39^{\circ} \mathrm{C}$, tachycardia, mild icterus and hepatosplenomegaly.

Laboratory analysis showed increased parameters of acute inflammation, liver and kidney function tests (Table 1). Magnetic resonance cholangiopancreatography (MRCP) revealed hepatosplenomegaly, multiple cysts in both kidneys with reduced parenchyma, and diffuse cystic/ fusiform dilatation of the intrahepatic bile ducts, which was more evident in the left lobe (Figures 1 and 2).

The patient had the first episode of pyelonephritis at 9 months of age, when vesicoureteral reflux and enlarged kidneys were also observed. Monitoring by a nephrologist started when patient was at age 20, due to chronic renal insufficiency assumed as a consequence of chronic pyelo-nephritis. Since then, he has been on a conservative treatment for chronic renal insufficiency.

During his thirties, he was treated in hospital several times for recurrent cholangitis. Magnetic resonance

Table 1. Laboratory data of the proband on admission to the hospital.

\begin{tabular}{|l|r|c|}
\hline Parameters & M-41 & $\begin{array}{c}\text { Reference } \\
\text { Ranges }\end{array}$ \\
\hline $\mathrm{Hb}(\mathrm{g} / \mathrm{dL})$ & 13.0 & $13.0-17.0$ \\
\hline $\mathrm{WBC}\left(10^{9} / \mathrm{L}\right)$ & 14.71 & $4.00-10.00$ \\
\hline Platelets $\left(10^{9} / \mathrm{L}\right)$ & 11.9 & $15.0-40.0$ \\
\hline Urea $(\mathrm{mmol} / \mathrm{L})$ & 12.6 & $3.2-7.4$ \\
\hline Creatinine $(\mu \mathrm{mol} / \mathrm{L})$ & 338.0 & $62.0-106.0$ \\
\hline Albumin $(\mathrm{g} / \mathrm{L})$ & 38.0 & $35.0-52.0$ \\
\hline ALP $(\mathrm{IU} / \mathrm{L})$ & 105.0 & $<129.0$ \\
\hline ALT $(\mathrm{IU} / \mathrm{L})$ & 178.0 & $<41.0$ \\
\hline ASP $(\mathrm{IU} / \mathrm{L})$ & 99.0 & $<37.0$ \\
\hline GGT $(\mathrm{IU} / \mathrm{L})$ & 127.0 & $<60.0$ \\
\hline Total bilirubin $(\mu \mathrm{mol} / \mathrm{L})$ & 22.0 & $<20.0$ \\
\hline CRP $(\mathrm{mg} / \mathrm{L})$ & 326.0 & $<5.0$ \\
\hline Fibrogen $(\mathrm{g} / \mathrm{L})$ & 4.6 & $1.8-4.0$ \\
\hline ESR $(\mathrm{mm} / \mathrm{h})$ & 40.0 & $1.0-10.0$ \\
\hline
\end{tabular}

Hb: hemoglobin; WBC: white blood cell count; ALP: alkaline hosphatase; ALT: alanine aminotransferase; ASP: aspartate aminotransferase; GGT: $\gamma$-Glutamyl transferase; CRP: C-reactive protein;

ESR: erythrocyte sedimentation rate.

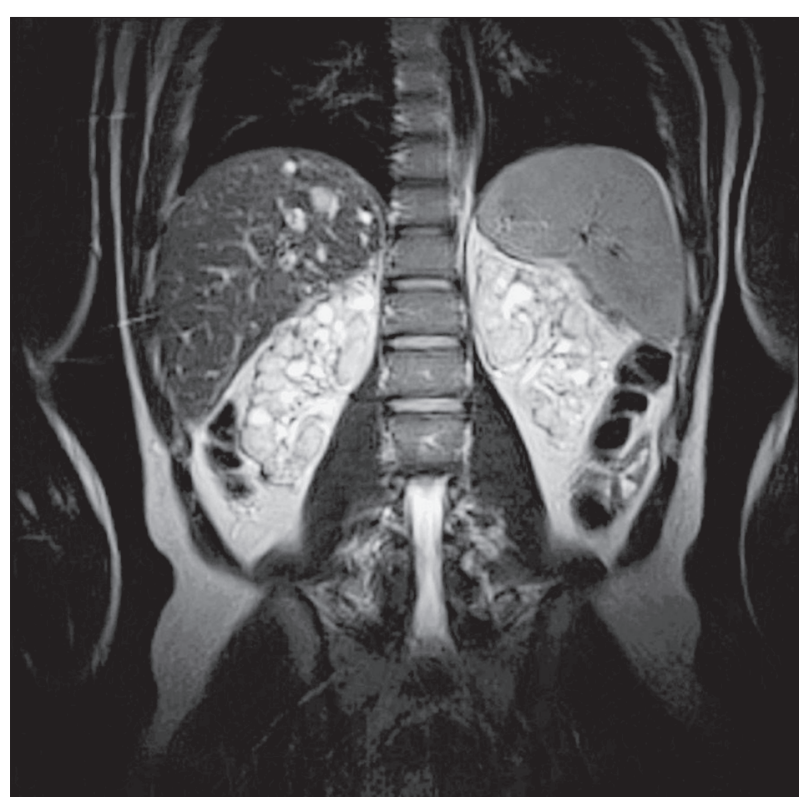

Figure 1. Magnetic resonance cholangiopancreatography of the frontal section: diffuse cystic/fusiform dilatation of the intrahepatic bile ducts, more in the left lobe, with enlarged polycystic kidneys.

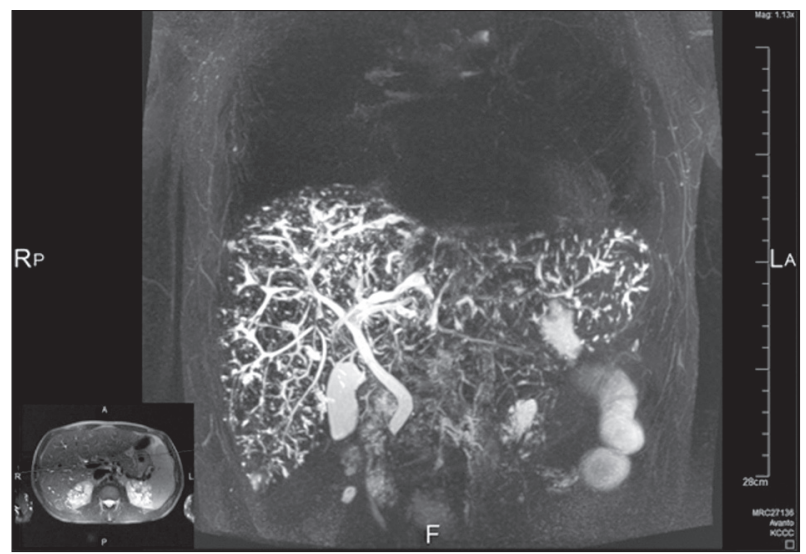

Figure 2. Magnetic resonance cholangiopancreatography reconstruction of the biliary tree: multiple cystic dilatations communicating with intrahepatic biliary tree and normal caliber of common choledochal duct.

chol-angiopancreatography and endoscopic retrograde chol-angiopancreatography (ERCP) were performed multiple times. First, the differential diagnosis of $\mathrm{CD}$ with polycystic kidney disease and PSC with dysplastic kidney disease was established. As part of further examination, a liver biopsy showed the existence of a multiple bile duct hamartomas $(\mathrm{MBH})$, without evidence of CHF and portal hypertension. On the following ERCP and MRCP examinations, changes in bile duct pointing to PSC were described and the diagnosis was leaning towards PSC. Thus, the diagnosis remained unclear after classical imaging studies and additional evidence was required to establish the definitive diagnosis. 


\section{MATERIALS AND METHODS}

In order to resolve the diagnostic dilemma (PSC or $\mathrm{CD}$ ), we referred the patient for genetic diagnostics, where clinical exome sequencing (CES) using a TruSight One capture kit (Illumina, San Diego, CA, USA) was performed, followed by sequencing on an Illumina MiSeq platform. The bioinformatic analysis was performed according to GATK Best Practices workflow [14,15]. The strategy for exome data interpretation was primarily based on the combined disease and phenotype gene target definition approach we previously described [16]. Considering the observation of multiple renal cysts in the patient, bioinformatic analysis and interpretation of genes associated with polycystic kidney disease was performed $(B I C C 1$, HNF1B, LRP5, NOTCH2, PKD1, PKD2, PKHD1, REN, SEC63, VHL).

Sequencing Results. Sequencing data analysis showed the presence of two pathogenic heterozygous variants in the $P K H D 1$ gene: nonsense variant c. $370 \mathrm{C}>\mathrm{T}$, corresponding to an amino acid change Arg124Ter and missense variant c. $4870 \mathrm{C}>\mathrm{T}$ corresponding to an amino acid change Arg1624Trp. Both identified variants are rare in the control population and in the 138,000 controls of the gnomAD project $[17,18]$, its population frequency was estimated at $0.0016 \%$ for $\mathrm{c} .370 \mathrm{C}>\mathrm{T}$ and $0.018 \%$ for c. $4870 \mathrm{C}>\mathrm{T}$, respectively. Based on the anticipated effect, the c.370C $>\mathrm{T}$ variant likely affects function of the protein coded by the gene with this variant, whereas for c. $4870 \mathrm{C}>\mathrm{T}$, it was predicted as pathogenic with two from the three pathogenicity prediction algorithms employed (Sift) [19,20], Polyphen2 [21,22], MutationTaster [23,24]. Both identified variants are featured in the ClinVar database as pathogenic (accession numbers: 167499; 188369) [25]. The reported variants in the PKHD1 gene present a likely cause for the clinical presentation of the patient. Based on recurrent cholangitis, cystic dilatation of the intrahepatic bile ducts associated with polycystic kidney disease, normal findings on extra hepatic bile ducts, presence of two pathogenic heterozygous variants in the PKHDl gene, we concluded that the correct diagnosis in this case is $\mathrm{CD}$.

\section{DISCUSSION}

In the presented patient, we discovered two pathogenic heterozygous variants in the PKHDI gene (c.370C $>\mathrm{T}$ and c. $4870 \mathrm{C}>\mathrm{T}$ ). Both variants have previously been reported as pathogenic in at least four patients with clinical presentation of ARPKD [26]. Furthermore, the rarity of the variants in the control population and the compatibility with clinical presentation, both favor the pathogenic nature of the identified variants. In accordance with guidelines for interpretation of sequence variants [27], these variants are classified as pathogenic, and present a likely cause for the clinical presentation of $\mathrm{CD}$ in the patient.

Two types of CD have been described in the literature, based on the presence of congenital hepatic fibrosis and the severity of the liver impairment. In our patient, CD presented clinically with recurrent cholangitis, which is normally the most common clinical manifestation of the type 1 disease, simply referred to as CD. Other clinical presentation of $\mathrm{CD}$ are varied and include right upper quadrant pain, jaundice, recurrent chills and fevers, weight loss, anorexia, nausea and vomiting, most of which were common complaints during several hospitalizations of the patients. On the other hand, CS is a complex clinical syndrome that includes characteristics of $\mathrm{CD}$ and $\mathrm{CHF}$, portal hypertension, liver cirrhosis, esophageal varices and ascites. Caroli's syndrome is mostly comorbid with cystic kidney diseases, and rarely with pancreatic or lineal cysts. Caroli's syndrome may progress to cholangiocarcinoma due to cirrhosis, chronic inflamation and prolonged exposure of the ductal epithelium to high concentration of un-conjugated secondary bile acids. Hepatobiliary malignant transformation occurs in approximately $7.0-14.0 \%$ of cases, and occurrence of cholangiocarcinoma in $\mathrm{CD} / \mathrm{CS}$ is 100 times greater than that in the general population. However, the division may be arbitrary, as many investigators believe that the two conditions present a continuation of the same disease. Nevertheless, the diagnosis in this patient established after clinical exome sequencing was $\mathrm{CD}$, due to the absence of the hepatic symptoms characteristic of CS. The mild clinical presentation bearing similarities to PSC, contributed to the difficulty of diagnosis in the presented patient as multiple MRCP and ERCP as well as liver biopsy, were insufficient to clearly diagnose $\mathrm{CD}$. In selected groups of patients genetic analysis may thus be helpful to differentiate between genetic and non genetic etiologies, as in our example, where clinical exome sequencing enabled the diagnosis of $\mathrm{CD}$ in this patient after the detection of the pathogenic variants in the PKHDl gene [11].

\section{CONCLUSIONS}

We report the discovery of two pathogenic variants on the PKHD1 gene, causing CD with polycystic kidney disease in a patient undiagnosed for many years. In selected cases, clinical testing may be insufficient to clearly differentiate between the genetic and non genetic etiologies of the observed disorders, as was in this case of CD. In this small group of patients, genetic diagnostics may prove helpful. Finally, in children and adult patients who present with recurrent cholangitis and hepato/splenomegaly or in all cases 
with cholangitis and polycystic kidney disease, a diagnosis of the genetic condition CD or CS, should be considered.

Declaration of Interest. The authors report no conflicts of interest. The authors alone are responsible for the content and writing of this article.

\section{REFERENCES}

1. Caroli J, Soupault R, Kossakowski J, Plocker L, Paradowska. [Congenital polycystic dilation of the intrahepatic bile ducts; attempt at classification]. Sem Hop. 1958; 34(8/2): 488-495/SP. [French].

2. Giovanardi RO. Monolobar Caroli's disease in an adult. Case report. Hepatogastroenterology. 2003; 50(54): 2185-2187.

3. Steinbrück K, Enne M, Fernandes R, Martinho JM, Pacheco-moreira LF. Living donor liver transplantation for Caroli's disease: A report of two cases. ISRN Surg. 2011; 2011): 106487-106490.

4. Yonem O, Bayraktar Y. Clinical characteristics of Caroli's disease. World J Gastroenterol. 2007; 13(13): 1930-1933.

5. Gu D, Park M, Jung C, Yoo Y, Cho J, Ryu H, et al. Caroli's disease misdiagnosed as intraductal papillary neoplasm of the bile duct. Clin Mol Hepatol. 2015; 21(2): 175-179.

6. Wang Z, Li Y, Wang R, Li Y, Li Z, Xiao X, et al. Clinical classification of Caroli's disease: An analysis of 30 patients. HPB (Oxford). 2015; 17(3): 278-283.

7. Caroli J. Diseases of the intrahepatic biliary tree. Clin Gastroenterol. 1973; 2(1): 147-161.

8. Alzarka B, Morizono H, Bollman J, Kim D, GuayWoodford L. Design and implementation of the hepatorenal fibrocystic disease core center clinical database: A centralized resource for characterizing autosomal recessive polycystic kidney disease and other hepatorenal fibrocystic diseases. Front Pediatr. 2017; 5: 80-85.

9. Shenoy P, Zaki S, Shanbag P, Bhongade S. Caroli’s syndrome with autosomal recessive polycystic kidney disease. Saudi J Kidney Dis Transpl. 2014; 25(4): 840-843.

10. Acioli ML, Costa LRG, de Miranda Henriques MS. Diffuse Caroli's disease with atypical presentation: A case report. Ann Gastroenterol. 2014; 27(1): 79-81.

11. Hao X, Liu S, Dong Q, Zhang H, Zhao J, Su L. Whole exome sequencing identifies recessive PKHD1 mutations in a Chinese twin family with Caroli disease. PLoS One. 2014; 9(4): e92661-e92666.
12. Courcet J, Minello A, Prieur F, Morisse L, Phelip J, Faivre L, et al. Compound heterozygous PKHD1 variants cause a wide spectrum of ductal plate malformations. Am J Med Genet A. 2015; 167A(12): 3046-3053.

13. Chung EM, Conran RM, Schroeder JW, RohenaQuinquilla IR, Rooks VJ. From the radiologic pathology archives: Pediatric polycystic kidney disease and other ciliopathies: radiologic-pathologic correlation. Radio-graphics. 2014; 34(1): 155-178.

14. McKenna A, Hanna M, Banks E, Sivachenko A, Cibulski K, Kernytsky A, et al. The genome analysis toolkit: A MapReduce framework for analyzing next-generation DNA sequencing data. Genome Res. 2010; 20(9): 1297-1303.

15. Broad Institute, Genome Analysis Toolkit: Variant discover in high-throughput sequencing data. Accessed December 2018 (https://software.broadinstitute.org.gatk/).

16. Maver A, Lovrečić L, Volk M, Rudolf G, Writzl K, Blatnic A, et al. Phenotype-driven gene target definition in clinical genome-wide sequencing data interpretation. Genet Med. 2015; 18(11): 1102-1110.

17. Lek M, Karczewski KJ, Minikel EV, Samocha KE, Banks E, Fennell T, et al. Analysis of protein-coding genetic variations in 60,706 humans. Nature. 2016; 536(7616): 285-291.

18. The Genome Aggregation Database. GnomAD. Accessed December 2018 (https:/gnomad.broadinstitute. org.about).

19. Sim NL, Kumar P, Hu J, Henikoff S, Schneider G, Ng PC. SIFT web server: Predicting effects of amino acid substitutions on proteins. Nucleic Acids Res. 2012; 40(Web Server issue): W452-W457.

20. Ng PC. Sorting intolerant from tolerant. Accessed December 2018 (https://sift.bii.a-star.edu.sg/).

21. Adzhubei IA, Schmidt S, Peshin L, Ramensky VE, Gerasimov A, Bork P, et al. A method and server for predicting damaging missense mutations. Nat Methods. 2010; 7(4): 248-249.

22. BWH Genetics, Harvard. PolyPhen-2: Prediction of functional effects of human nsSNPs. Accessed December 2018 (https://genetics.bwh.harvard.edu/ pph2/).

23. Schwarz JM, Cooper DN, Schuelke M, Seelow D. MutationTaster2: Mutation prediction for the deepsequencing age. Nat Methods. 2014; 11(4): 361-362.

24. Seelow D. Mutation Taster. Accessed December 2018 (http://www.mutationtaster.org/). 
25. Landrum MJ, Lee JM, Riley GR, Jang W, Rubinstein WS, Church DM, et al. ClinVar: Public archive of relationships among sequence variation and human phenotype. Nucleic Acids Res. 2014; 42(Database issue): D980-D985.

26. Melchionda S, Palladino T, Castellana S, Giordano M, Benetti E, De Bonis P, et al. Expanding the mutation spectrum in 130 probands with ARPKD: Identification of 62 novel PKHD1 mutations by Sanger sequencing and MLPA analysis. J Hum Genet. 2016; 61(9): 811-821.
27. Richards S, Aziz N, Bale S, Bick D, Das S, GastierFoster J, et al.; ACMG Laboratory Quality Assurance Committee. Standards and guidelines for the interpretation of sequence variants: A joint consensus recommendation of the American College of Medical Genetics and Genomics and the Association for Molecular Pathology. Genet Med. 2015; 17(5): 405-424. 